Vo1. 11 (1988)

\title{
ABSTRACT KINETIC EQUATIONS WITH ACCRETIVE COLLISION OPERATORS
}

\author{
Alexander H. Ganchev ${ }^{1}$, William Greenberg ${ }^{2}$ and C.V.M. van der Mee ${ }^{3}$
}

We consider the class of abstract kinetic equations $(T \psi)^{\prime}(x)=-A \psi(x)$ on the half-line $x \in(0, \infty)$ where $T$ is an injective self-adjoint operator and $A$ is an accretive compact perturbation of the identity, both of them defined on Hilbert space. Half-range boundary conditions are imposed. If $\operatorname{Re} A>\delta 1$ for some $\delta>0$, we establish the unique solvability of the problem. If $\operatorname{Re} A \geq 0$ and $\operatorname{Ker} A=$ $\operatorname{Ker}(\operatorname{Re} A$ ), we prove that the problem has at least one bounded (in norm) solution and give a complete description of its measure of nonuniqueness. The result is applied to derive the well-posedness of the equation of transfer of polarized light and some multigroup neutron transport equations.

\section{INTRODUCTION}

Since the seminal work of Hangelbroek and Lekkerkerker $[H, H L]$ on the subcritical neutron transport equation in a homogeneous half-space with isotropic scattering much effort has been spent in constructing a complete existence and uniqueness theory of abstract kinetic equations of the type

$$
\begin{aligned}
& (T \psi)^{\prime}(x)=-A \psi(x), \quad 0<x<\infty, \\
& Q_{+} \psi(0)=\varphi_{+}, \\
& \|\psi(x)\|_{H}=O(1) \quad(x \rightarrow \infty)
\end{aligned}
$$

\footnotetext{
${ }^{1}$ Research conducted as part of a Ph.D. thesis accepted by Virginia Tech.

2 Research supported in part by the U.S. Dept. of Energy under grant No. DE-FG05-97ER25033 and the National Science Foundation under grant No. DMS 8312452.

${ }^{3}$ Research supported by the National Science Foundation grant DMS 8501337.
} 
where $T$ is an injective self-adjoint operator on a complex Hilbert space $H, Q_{+}$is the orthogonal projection of $H$ onto the maximal subspace on which $\left\langle T_{\cdot \cdot}\right\rangle$ is positive and $\mathrm{A}$ is some operator on $\mathrm{H}$. Roughly speaking, there are two families of abstract kinetic theories, each pertaining to different types of operators $T$ and $A$.

One of these theories was developed to a large extent by Beals. In this method one extends the given Hilbert space setting and seeks the solution in the completion, $\mathrm{H}_{\mathrm{T}}$, of the domain of $\mathrm{T}, \mathrm{D}(\mathrm{T})$, with respect to the inner product \langle| $\mathrm{TI}$, , $\rangle$. It can be applied succesfully when $A$ is nonnegative bounded self-adjoint with the null space of $A$, Ker A, finite-dimensional (see [B1]), or when $A$ is a nonnegative self-adjoint Sturm-Liouville differential operator and $T$ is the multiplication by an indefinite weight function (see [B2]).

The other branch of abstract kinetic theory deals with operators A which are compact perturbations of the identity. Here no extension of the solution space from $\mathrm{H}$ to $\mathrm{H}_{\mathrm{T}}$ is needed. In fact, the method relies on an application of the Fredholm alternative such as the one carried out in [HL] for neutron transport with isotropic scattering. Using this method Van der Mee [M1] settled the well-posedness issue when $T$ is bounded and $A$ is a nonnegative self-adjoint compact perturbation of the identity. The result was subsequently generalized by Greenberg et al. [GMW] to the case when $T$ is unbounded. In these publications the selfadjointness properties of $T^{-1}$ and $T^{-1} A$ were used to prove a certain decomposition of $H$ which is equivalent to the unique solvability of Eqs. (1.1)-(1.3). Here, as in the method used by Beals, the positive self-adjointness of A plays a seemingly essential role.

The consideration of operators $A$ which are compact perturbations of the identity allows one to prove the equivalence of Eqs. (1.1)-(1.3) to a vector-valued Wiener-Hopf equation of the form

$$
\psi(x)-\int_{0}^{\infty} J \mathcal{G}(x-y) B \psi(y) d y=\omega(x), 0<x<\infty
$$

where $B=I I-A$ is a compact operator,

$$
\mathcal{H}(z)=\left\{\begin{array}{cc}
+\int_{0}^{\infty} \mathrm{t}^{-1} \mathrm{e}^{-z / t} \sigma(d \mathrm{t}), & z>0 \\
-\int_{-\infty}^{0} \mathrm{t}^{-1} \mathrm{e}^{-z / t} \sigma(d t), & z<0
\end{array}\right.
$$

and $\omega(x)=\int_{0}^{\infty} e^{-x / t} \sigma(d t) \varphi_{+}$. Here $\varphi_{+} \in Q_{+}[D(T)]$ and $\sigma(\cdot)$ denotes the resolution of the identity of $T$. The equivalence of the boundary value problem (1.1)-(1.3) and 
the vector-valued Wiener-Hopf equation (1.4) under the minor regularity condition

$$
\exists \alpha>0, \beta>1: \operatorname{Ran} \mathrm{B} \subseteq \operatorname{Ran}|\mathrm{T}|^{\alpha} \cap \mathrm{D}\left(|\mathrm{TT}|^{\beta}\right)
$$

was in fact established by Van der Mee [M1,M3]. Here and in the sequel Ran S denotes the range and $D(S)$ the domain of an operator $S$. It turns out that the operator governing the left-hand side of Eq. (1.4) is a Fredholm operator on one of (and hence each one of) the Banach spaces $L_{p}(H)_{0}^{\infty}(1 \leq p \leq \infty)$ of Bochner $L_{p}$-integrable, $\mathrm{C}(\mathrm{H})_{0}^{\infty}$ of bounded strongly continuous functions $w:[0, \infty) \rightarrow \mathrm{H}$ and $\mathrm{C}_{0}(\mathrm{H})_{0}^{\infty}$ of bounded strongly continuous functions $\psi:[0, \infty]-H$ with $\psi(\infty)=0$, provided $T^{-1} A$ does not have zero or purely imaginary eigenvalues, irrespective of whether $A$ is selfadjoint or not. This opens the way to proving the well-posedness of Eqs. (1.1)-(1.3) for certain classes of non-selfadjoint $A$ by taking the following path:

(i) to prove that Eqs. (1.1)-(1.3) with $\varphi_{+}=0$ have the zero solution only,

(ii) to prove that the operator governing the left-hand side of Eq. (1.4) is a Fredholm operator of index zero, and

(iii) to exploit the Fredholm alternative and the equivalence of Eqs. (1.1)-(1.3) and Eq. (1.4) to obtain the unique solvability of Eqs. (1.1)-(1.3)

This path, a key observation was made by Willis et al. [WZM], was taken before when studying Eq. (1.1) with $x$ in the bounded interval $(0, \tau)$ where $T$ is injective self-adjoint and $\operatorname{Re} A \geq 0$ with $\operatorname{Ker} A=\operatorname{Ker}(\operatorname{Re} A)$. However, the fact that in this case the boundary value problem (1.1)-(1.3) is equivalent to a vector-valued convoIution equation on the finite interval $(0, \tau)$ and this convolution operator is known to be compact, settled step (ii) in an almost trivial manner. When working on the half-line $(0, \infty)$, however, the second step of the above procedure is more difficult to implement.

Many of the topics sketched above may be found in two monographs. The first one, by Kaper et al. [KLH], deals mainly with applications in one-speed neutron transport theory and emphasizes the expansion of the solution with respect to the (singular) eigenfunctions of the evolution operator $\mathrm{T}^{-1} \mathrm{~A}$, which can be done rigorously if $\mathrm{A}$ is positive self-adjoint. The second monograph, by Greenberg et al. [GMP], gives a theory of abstract boundary value problems of the type (1.1)-(1.3) and vector-valued convolution equations of the form (1.4) and applies it to a host of applications in neutron transport theory, radiative transfer, rarefied gas dynamics and other fields. In [GMP] a number of problems with non-selfadjoint $A$ was treated by first developing some bisemigroup perturbation theory and then apply- 
ing this theory to the problem under consideration. One of the cases considered was the situation in which $A$ has a positive real part, which were actually results obtained by Ganchev et al. (see [Ga,GaG], also [GaGM]). In this article we supply a new proof of these results which does not hinge on the rather cumbersome construction of the analytic bisemigroup generated by $T^{-1} A$. Instead we follow the path described above and circumvent the problem of how to define certain projections and semigroups altogether.

In Section 2 we settle the case when $\operatorname{Re} A \geq \delta 11$ for some $\delta>0$. In Section 3 we extend our results to the case when $\operatorname{Re} A \geq 0$ and $\operatorname{Ker} A=\operatorname{Ker}(\operatorname{Re} A) \geq 0$, while Sections 4 and 5 are devoted to applications and a discussion.

\section{STRICTLY ACCRETIVE COLLISION OPERATORS}

Throughout this section $T$ will be an injective self-adjoint operator and $A$ a compact perturbation of the identity satisfying $\operatorname{Re} A \geq \delta$ II for some $\delta>0$, both of them defined on the complex Hilbert space $H$. We will assume that condition (1.6) holds true. The effect of condition (1.6) will be that the operator function $36(.) \mathrm{B}$ occurring as the convolution kernel in Eq. (1.4) as well as the operator function TYG(.)B are Bochner integrable functions from $R$ into $L(H)$, the Banach algebra of bounded linear operators on $H$. As a result, the convolution operators

$$
\left(\mathcal{L}_{\mathrm{B}} \psi\right)(\mathrm{x})=\int_{0}^{\infty} \mathscr{H}(\mathrm{x}-\mathrm{y}) \mathrm{B} \psi(\mathrm{y}) \mathrm{dy}, \quad\left(\mathcal{L}_{\mathrm{TB}} \psi\right)(\mathrm{x})=\int_{0}^{\infty} \mathfrak{H}(\mathrm{x}-\mathrm{y}) \mathrm{TB} \psi(\mathrm{y}) \mathrm{dy}
$$

are bounded on $E(H)_{0}^{\infty}$ where $E(H)_{0}^{\infty}$ denotes one of the spaces $L_{p}(H)_{0}^{\infty}(1 \leq p \leq \infty)$, $\mathrm{C}(\mathrm{H})_{0}^{\infty}$ and $\mathrm{C}_{0}(\mathrm{H})_{0}^{\infty}$ defined in the introduction. Moreover, if $\psi \in E(H)_{0}^{\infty}$, then $\left(\ell_{B} \psi\right)(x) \in D(T)$ for almost every (and, if $E(H)_{0}^{\infty}=C(H)_{0}^{\infty}$ or $C_{0}(H)_{0}^{\infty}$, every) $x \in[0, \infty)$ and $T\left(\mathcal{L}_{\mathrm{B}} \not\right)(\mathrm{x}) \equiv\left(\mathcal{L}_{\mathrm{TB}} \not\right)(\mathrm{x})$. The necessary background information on convolution operators can be found in [GK] (generalized in [Fe3] to an infinite-dimensional setting) and in Chapters VI and VII of [CMP]. We will go through the three steps pointed out in the introduction to establish the unique solvability of Eqs. (1.1)(1.3).

LEMMA 2.1. Let $\mathrm{T}$ be an injective selt-adjoint operator on $\mathrm{H}$, and let $\varphi_{+} \in \mathrm{Q}_{+}[\mathrm{H}]$. Then the vector function $\omega:[0, \infty) \rightarrow \mathrm{H}$ defined in the introduction belongs to $\mathrm{L}_{2}(\mathrm{H})_{0}^{\infty}$ if and only if $\varphi_{+} \in \mathrm{D}\left(\mid \mathrm{TT}^{1 / 2}\right)$. 
Proof: Using the Spectral Theorem we find that $\int_{0}^{\infty}\|\omega(x)\|_{\mathrm{H}}^{2} \mathrm{dx}=\frac{1}{2}\left\|\left.\mathrm{~T}\right|^{1 / 2} \varphi_{+}\right\|_{\mathrm{H}}$, which establishes the lemma.

LEMMA 2.2. Under the general hypotheses of this sectlon, there is at most one solution of Eqs. (1.1)-(1.3).

Proof: Suppose $\psi$ is a solution of Eqs. (1.1)-(1.3) with $\varphi_{+}=0$. Then, by definition, (t) $[0, \infty] \rightarrow H$ is a bounded (in $H$ ) strongly continuous vector function such that $T(x)$ $\in D(T)$ for all $x \in(0, \infty), T \psi$ is strongly differentiable with derivative $-A \psi$ and $Q_{+} \psi(0)=0$. Thus $\psi$ belongs to the null space of $I I-L_{B}$ in $C(H)_{0}^{\infty}$ and therefore to its null space in $L_{2}(H)_{0}^{\infty}$, because the Fredholm characteristics of $11-L_{B}$ are independent of the particular choice of $E(H)_{0}^{\infty}$. Let us write $Q_{-}=1 I-Q_{+}$; then $Q_{-}$ is the orthogonal projection of $H$ onto the maximal subspace on which $\langle\mathrm{T} \cdot \cdot\rangle$ is negative. Using that $\operatorname{Re} A \geq 0$, we easily compute

$$
\begin{aligned}
& 0 \geq-2 \int_{0}^{\infty}\left\langle(\operatorname{ReA}) \psi(\mathrm{x}), \psi(\mathrm{x})>\mathrm{dx}=-\int_{0}^{\infty}[\langle\mathrm{A} \psi(\mathrm{x}), \psi(\mathrm{x})\rangle+\langle\psi(\mathrm{x}), \mathrm{A} \psi(\mathrm{x})\rangle] \mathrm{dx}=\right. \\
& =\int_{0}^{\infty} \frac{\mathrm{d}}{\mathrm{dx}}\left\langle\mathrm{T} \psi(\mathrm{x}), \psi(\mathrm{x})>\mathrm{d} \mathrm{x}=-\langle\mathrm{T} \psi(0), \psi(0)\rangle+\lim _{\tau \rightarrow \infty}\langle\mathrm{T} \phi(\tau), \psi(\tau)\rangle\right.
\end{aligned}
$$

where the limit is easily seen to exist. In fact, this limit varishes. In order to see this, we note that $\langle T \psi(\cdot), \psi(\cdot)\rangle \in L_{1}\left(R_{+}\right)$, because the vectors $\psi \in L_{2}\{H)_{0}^{\infty}, T(\psi-\omega)=$

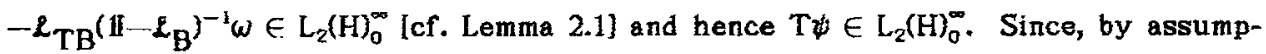
tion, $Q_{+} \psi(0)=0$, we obtain

$$
0 \geq-2 \int_{0}^{\infty}<(\operatorname{ReA}) \psi(x), \psi(x)>d x=-\langle T \psi(0), \psi(0)>\geq 0
$$

But the integrand in the last expression is nonnegative and $\operatorname{Re} A \geq 0$, so that

$$
(\operatorname{Re} A) \psi(x)=0
$$

Because $\operatorname{Re} A \geq \delta 1$ for some $\delta>0$, we conclude that $\downarrow=0$, as claimed. $\square$

In order to establish the next lemma, we make two observations. First, if $\operatorname{Re} A \geq \delta 11$ for some $\delta>0$, then $T^{-1} A$ cannot have any zero or purely imaginary eigenvalues. Indeed, if $A \xi=\lambda T \xi$ for some $\lambda$ with $\operatorname{Re} \lambda=0$, then 


$$
((\operatorname{Re} A) \xi, \xi)=(\lambda T \xi, \xi)+(\xi, \lambda T \xi)=\lambda(T \xi, \xi)+\bar{\lambda}(T \xi, \xi)=\lambda\{(T \xi, \xi)-(\xi, T \xi)\}=0,
$$

whence $0 \leq \delta\|\xi\|^{2} \leq(\{\operatorname{Re} A) \xi, \xi)=0$ and thus $\xi=0$. The second observation, due to Feldman [Fe], is that the convolution operator

$$
\left(\mathcal{W} \psi(x)=\int_{0}^{\infty} \mathfrak{H}(x-y) \psi(y) d y\right.
$$

is bounded on $\mathrm{L}_{2}(\mathrm{H})_{0}^{\infty}$ with unit norm. In fact, $\mathcal{N}$ is a projected restriction of the operator $\left(\mathcal{N}_{ \pm} \psi\right)(x)=\int_{-\infty}^{\infty} J 6(x-y) \psi(y) d y$ on the Hilbert space $L_{2}(H)_{-\infty}^{\infty}$ of strongly measurable $\mathrm{L}_{2}$-functions $\psi: R \rightarrow H$, which is unitarily equivalent (through the Fourier transform) to the operator $\left(\tilde{\mathcal{N}}_{\perp} \psi\right)(\mathrm{x})=(\mathrm{II}-\mathrm{ixT})^{-1} \psi(\mathrm{x})$ defined on $\mathrm{L}_{2}(\mathrm{H})_{-\infty}^{\infty}$ and the last operator has unit norm.

LEMMA 2.3. Under the general hypotheses of this section, the operator $11-L_{B}$ is boundedly invertible on any of the Banach spaces $\mathrm{E}(\mathrm{H})_{0}^{\infty}$.

Proof: Consider the Hilbert space $\mathrm{L}_{2}(\mathrm{H})_{-\infty}^{\infty}$. Then $\mathrm{J}=\operatorname{sgn}(\mathrm{T}), \mathrm{B}$ and its adjoint $\mathrm{B}^{*}$ can be viewed as bounded linear operators on $\mathrm{L}_{2}(\mathrm{H})_{-\infty}^{\infty}$; then $J$ is a unitary operator on $L_{2}(H)_{-\infty}^{\infty}$ such that $J^{2}=1$. Since $\mathcal{L}_{B}=\mathcal{N B}$ on $\mathrm{L}_{2}(\mathrm{H})_{-\infty}^{\infty}$, we easily obtain

$$
\mathcal{L}_{\mathrm{B}}^{*}=\mathrm{J} \cdot \mathrm{JB}^{*} \mathrm{~J} \cdot \mathcal{N} \cdot \mathrm{J},
$$

which is similar to the operator

$$
\left(\mathcal{L}_{C} \psi\right)(\mathrm{x})=\int_{0}^{\infty} \mathfrak{H}(x-y) C \psi(y) d y
$$

on $\mathrm{L}_{2}(\mathrm{H})_{-\infty}^{\infty}$ where $\mathrm{C}=\mathrm{JB}^{*} \mathrm{~J}$. Now note that both $11-\mathrm{B}$ and $\mathbb{1 l}-\mathrm{C}$ have their real part $\geq \delta 11$ for some $\delta>0$. According to Lemma 2.1, the equivalence of Eqs. (1.1)(1.3) and Eq. (1.4) on $\mathrm{C}(\mathrm{H})_{0}^{\infty}$ and the equality of the Fredholm characteristics of II $-L_{B}$ on $L_{2}(H)_{0}^{\infty}$ and $C(H)_{0}^{\infty}$, both for $A=1 I-B$ and for $J A^{*} J=1 I-C$, we may conclude that $11-L_{B}$ and $11-L_{C}$ have zero null space. However, the latter operator is similar to the adjoint of the former while both of them have closed range. Thus both of these operators are invertible on $L_{2}(H)_{0}^{\infty}$.

Finally, since the Fredholm characteristics of $11-L_{B}$ are the same on any of the spaces $E(H)_{0}^{\infty}$ mentioned above, we find $I I-L_{B}$ to be invertible on each one 
of the spaces $\mathrm{E}(\mathrm{H})_{0}^{\infty}$.

THEOREM 2.4. Under the general hypotheses of this section, the boundary-value problem (1.1)-(1.3) has a unlque solution $\psi:[0, \infty) \rightarrow H$ for every $\varphi_{+} \in Q_{+}[D(T)]$, which satisfies both of the conditions $\|\psi(x)\|_{\mathrm{H}}=o(1)$ and $\|\mathrm{T} \psi(\mathrm{x})\|_{\mathrm{H}}=\mathrm{o}(1) \quad(\mathrm{x} \rightarrow \infty)$.

Proof: From the above lemmas and the equivalence of Eqs. (1.1)-(1.3) and Eq. (1.4) on $\mathrm{C}(\mathrm{H})_{0}^{\infty}$ it is immediate that for every $\varphi_{+} \in \mathrm{Q}_{+}[D(T)]$ there exists a unique bounded (in $H$ ) strongly continuous vector function $\psi:(0, \infty) \rightarrow H$ such that $\psi(x) \in$ $D(T)$ for all $x \in(0, \infty), T \psi$ is strongly differentiable on $(0, \infty)$ with derivative $-\mathrm{A} \psi(\mathrm{x})$ and $\mathrm{Q}_{+} \psi(0)=\varphi_{+}$. Furthermore, the invertibility of $11-L_{\mathrm{B}}$ on $\mathrm{C}_{0}(\mathrm{H})_{0}^{\infty}$ and $\omega \in C_{0}(H)_{0}^{\infty}$ imply $\|\psi(x)\|_{H}=o(1)(x \rightarrow \infty)$. Since $\varphi_{+} \in D(T)$ and $T(\psi-\omega)=$ ${ }^{-\mathcal{L}_{T B}}\left(11-\mathcal{L}_{\mathrm{B}}\right)^{-1} \omega$, we also obtain $\|\mathrm{T} \downarrow(\mathrm{x})\|_{\mathrm{H}}=\mathrm{o}(1)(\mathrm{x} \rightarrow \infty)$, which completes the proof. 口

Under the assumptions of Theorem 2.4, we have in fact $\psi(0) \in D(T)$ whenever $\varphi_{+} \in Q_{+}[D(T)]$. Indeed, since in this case $\{\omega, T \omega\} \subseteq C(H)_{0}^{\infty}$, we have $\psi \in$ $\mathrm{C}(\mathrm{H})_{0}^{\infty}$ and $\mathrm{T}(\psi-\omega)=-\mathcal{L}_{\mathrm{TB}}\left(11-\mathcal{L}_{\mathrm{B}}\right)^{-1} \omega \in \mathrm{C}(\mathrm{H})_{0}^{\infty}$ and hence $\mathrm{T} \psi \in \mathrm{C}(\mathrm{H})_{0}^{\infty}$, which implies $\not(0) \in D(T)$, as claimed above.

COROLLARY 2.5. Suppose condition (1.6) is satisfied as well as $\|\mathrm{II}-\mathrm{A}\|_{\mathrm{L}(\mathrm{H})}<1$. Then the boundary value problem (1.1)-(1.3) has a unique solution.

If is easily verified that, under the conditions of the corollary, $\operatorname{Re} A \geq \delta 1$ with $\delta=1-\|B\|>0$. In fact, as observed in [GMP], the corollary is also immediate from the equivalence of Eqs. (1.1)-(1.3) and Eq. (1.4) and the main result of [GL2] on factorization of operator functions close to the identity, since the symbol of the Wiener-Hopf equation (1.4), i.e. the operator function

$$
\tilde{W}(\lambda)=11-\int_{-\infty}^{\infty} e^{i x \lambda \lambda} \exists(x) B d x=11-(11-i \lambda T)^{-1} B
$$

satisfies

$$
\sup _{\lambda \in \mathbf{R}}\|11-\tilde{W}(\lambda)\|_{L(H)}<1
$$

For later use we introduce the following two complementary closed subspa- 
ces of $H$. By $H_{p}$ (resp. $H_{m}$ ) we denote the subspace of all vectors in $H$ which can be represented as $\psi\left(0^{ \pm}\right)$with $\psi$ being the solution of the Wiener-Hopf equation

$$
\psi(x) \mp \int_{0}^{ \pm \infty} \mathrm{J} 6(\mathrm{x}-\mathrm{y}) \mathrm{B}_{ \pm} \psi(\mathrm{y}) \mathrm{dy}= \pm \int_{0}^{+\infty} \mathrm{e}^{-\mathrm{x} / \mathrm{t}} \sigma(\mathrm{dt}) \varphi_{ \pm}
$$

in $\mathrm{C}(\mathrm{H})_{0}^{\infty}$ (resp. $\mathrm{C}(\mathrm{H})_{-\infty}^{\circ}$ ) for some $\varphi_{ \pm} \in \mathrm{Q}_{ \pm}[\mathrm{H}]$. Here $\mathrm{B}_{+}=\mathrm{B}$ and $\mathrm{B}_{-}=\mathrm{B}^{*}$. (The $\mathrm{u}-$ nique solvability of the equation on the left half-line is clear from the above results when replacing $T$ and $A$ with $-T$ and $A^{*}$.) Since the right-hand sides form a closed subspace, so do the solutions and hence the values of $\psi(0)$, whence $\mathrm{H}_{p}$ and $H_{m}$ are closed subspaces of $H$. Moreover, if $\varphi_{\perp} \in Q_{\perp}[D(T)]$ and $\psi_{\perp}$ are the corresponding half-space solutions, then

$$
\begin{aligned}
& \left\langle\mathrm{T} \psi_{+}\left(0^{+}\right), \psi_{-}\left(0^{-}\right)\right\rangle=-\int_{0}^{\infty} \frac{\mathrm{d}}{\mathrm{dx}}\left\langle\mathrm{T} \psi_{+}(\mathrm{x}), \psi_{-}(-\mathrm{x})\right\rangle \mathrm{dx}= \\
& =\int_{0}^{\infty}\left[\left\langle\mathrm{A} \psi_{+}(\mathrm{x}), \psi_{-}(-\mathrm{x})\right\rangle-\left\langle\psi_{+}(\mathrm{x}), \mathrm{A}^{*} \psi_{-}(-\mathrm{x})\right\rangle\right) \mathrm{dx}=0,
\end{aligned}
$$

which proves $H_{p} \cap D(T)$ and $H_{m} \cap D(T)$ to be orthogonal with respect to the indefinite inner product $\langle T,$,$\rangle . A similar calculation shows that for a non-trivial solu-$ tion $\psi_{ \pm}$

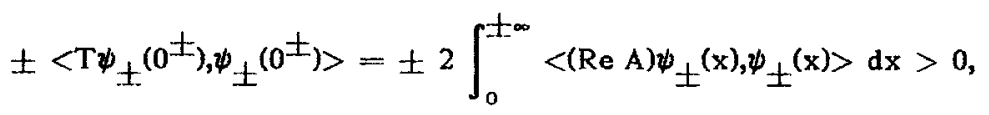

so that $H_{p} \cap D(T)$ is positive definite and $H_{m} \cap D(T)$ is negative definite with respect to the indefinite inner product $\langle\mathrm{T},$,$\rangle and hence have trivial intersection.$

It remains to prove $\mathrm{H}_{p}+\mathrm{H}_{m}=\mathrm{H}$. First note that (II - $\left.\mathbb{L}_{\mathrm{B}_{-}}^{(-)}\right) \oplus\left(11-\mathcal{L}_{\mathrm{B}_{+}}^{(+)}\right.$), the direct sum of the two operators pertaining to the left-hand sides of Eqs. $\left(2.2^{ \pm}\right)$, is invertible on each one of the Banach spaces $E(H)_{-\infty}^{\infty} \approx E(H)_{-\infty}^{0} \oplus E(H)_{0}^{\infty}$. Thus this operator maps the space of pairs of right-hand sides $\omega_{-} \oplus \omega_{+}$ bijectively onto the space of solution pairs $\psi_{-} \oplus \boldsymbol{\psi}_{+}$, so that $\mathrm{H}_{p}+\mathrm{H}_{m}$ is a closed subspace of $\mathrm{H}$. Its adjoint, when defined on $\mathrm{I}_{2}(\mathrm{H})_{-\infty}^{\infty}$, is obviously similar to (II $\left.-L_{\mathrm{B}_{+}}^{(-)}\right) \oplus\left(I I-\mathcal{L}_{\mathrm{B}_{\sim}}^{(+)}\right.$) on $\mathrm{L}_{2}(\mathrm{H})_{-\infty}^{\infty}$ [cf. the proof of Lemma 3 ], which has the analogous property. Hence, $\mathrm{H}_{p}+\mathrm{H}_{m}=\mathrm{H}$, as claimed.

We remark that $H_{p}$ and $H_{m}$ are invariant under $T^{-1} A$ and that the restrictions of $T^{-1} A$ to $H_{p}$ and $H_{m}$ have their spectra within the right and left half-plane, respectively. 


\section{NON-STRICTLY ACCRETIVE COLLISION OPERATORS}

In this section we will make the same assumptions on $T$ and $A$ as in Section 2 , except for the assumption $\operatorname{Re} A \geq \delta 11$, which will be replaced by the pair of hypotheses $\operatorname{Re} A \geq 0$ and $\operatorname{Ker} A=\operatorname{Ker}(\operatorname{Re} A)$, where $\operatorname{Ker} S$ denotes the null space of an operator $S$. We now define

$$
Z_{0}=\bigcup_{n=1}^{\infty} \operatorname{Ker}\left(T^{-1} A^{*}\right)^{n}
$$

We impose the extra condition on $T$ and $A$ that $\left.Z_{0} \subseteq D(I T)^{\beta}\right)$ for some $\beta>2$, which is fulfilled if (1.6) is valid for some $\alpha>0$ and $\beta>3$ [cf. Lemma 3.1(ii) below].

The subspace $Z_{0}$ was first considered in kinetic theory (in fact, conservative neutron transport with isotropic scattering) by Lekkerkerker [Le]. Below we will reduce the present boundary value problem to a boundary value problem with modified A satisfying $\operatorname{Re} A \delta \delta$ II for some $\delta>0$ and a finite-dimensional problem. Such a reduction was made before in $[\mathrm{M} 1, \mathrm{Be} 3, \mathrm{GMZ}]$. In dealing with the finite-dimensional problem, we will make frequent use of indefinite inner product spaces of finite dimension. For the theory of such spaces we refer to [Bo,GLR].

We have (cf. [M1,GMZ] for $A \geq 0$; [GMP] for $\operatorname{Re~} A \geq 0$ )

\section{LEMMA 3.1. The following statements hold true:}

(i) $\operatorname{Ker} \mathrm{A}=\operatorname{Ker} \mathrm{A}^{*}=\operatorname{Ker} \operatorname{Re} \mathrm{A}$ has finite dimension, $\mathrm{m}$ say,

(ii) $Z_{0}=\operatorname{Ker}\left(T^{-1} A\right)^{2}=\operatorname{Ker}\left(T^{-1} A^{*}\right)^{2}$ has finite dimension, $n$ say,

(iii) $\operatorname{Ker} \mathrm{A} \cap \operatorname{Ran}\left(\mathrm{T}^{-1} \mathrm{~A}\right)=\operatorname{Ker} \mathrm{A} \cap \operatorname{Ran}\left(\mathrm{T}^{-1} \mathrm{~A}^{*}\right)$ has finite dimension, namely $\mathrm{n}-\mathrm{m}$.

(iv) $Z_{0} \subseteq D(T)$,

(v) $\mathrm{T}^{-1} \mathrm{~A}$ does not have purely imeginary eigervalues.

Proof: Statement (i) is immediate from $\operatorname{Ker} A=\operatorname{Ker} \operatorname{Re} A$. Now suppose $A \xi=T \eta$, $A \eta=T S$ and $A S=0$. Then

$$
\begin{aligned}
& 2\langle(\operatorname{Re} \mathrm{A}) \eta, \eta\rangle=\langle\mathrm{A} \eta, \eta\rangle+\langle\eta, \mathrm{A} \eta\rangle=\langle\mathrm{T} S, \eta\rangle+\langle\eta, \mathrm{TS}\rangle= \\
& =\langle\mathrm{S}, \mathrm{A} \xi\rangle+\langle\mathrm{A} \xi, S\rangle=\left\langle\mathrm{A}^{*} \mathrm{~S}, \boldsymbol{\xi}\right\rangle+\left\langle\xi, \mathrm{A}^{*} \mathrm{~S}\right\rangle=0,
\end{aligned}
$$

by virtue of $(i)$, whence $T S=A \eta=(\operatorname{Re} A) \eta=0$ and thus $5=0$. As the argument 
with A replaced by $A^{*}$ is the same, we obtain (ii) from (i). The statement (iii) follows easily from (i) and $A$ and $A^{*}$ having closed range, while (iv) is immediate from (ii) and $\operatorname{Ker} A \subseteq D(T)$ [cf. (1.6)]. To derive (v), we simply repeat the calculation displayed in (2.1).

LEMMA 3.2. Consider the indefinite inner product $\left\langle\mathrm{T}_{9},\right\rangle$ on $\mathrm{Z}_{0}$. Then the following statements hold true:

(i) If $\xi \in Z_{0}$ and $\langle T \xi, \eta\rangle=0$ for all $\eta \in Z_{0}$, then $\xi=0$, l.e. $Z_{0}$ is non-degenerate,

(ii) $\mathcal{S G}_{0} \stackrel{\text { def. }}{=} \operatorname{Ker} \mathrm{A} \cap \operatorname{Ran}\left(\mathrm{T}^{-1} \mathrm{~A}\right)=\{\xi \in \operatorname{Ker} \mathrm{A}:\langle\mathrm{T} \xi, \eta>=0$ for $a l l \eta \in \operatorname{Ker} \mathrm{A}$, l.e. this subspace is the neutral part of Ker A,

(iii) If $\mathcal{N}_{ \pm}$is a maximal positive/negative definite subspace of Ker A, then $\mathfrak{M}_{0} \oplus$ $\mathcal{N}_{ \pm}$is a maximal positive/negative subspace of $Z_{0}$.

Proof: Suppose $\xi \in Z_{0}$ and $\langle T \xi, \eta\rangle=0$ for all $\eta \in Z_{0}$. Then certainly $\langle T \xi, \eta\rangle=$ 0 for all $\eta \in \operatorname{Ker} A^{*}$ and hence $T \xi \in\left[\operatorname{Ker} A^{*}\right]^{\perp}=\operatorname{Ran} A$. Now write $T \xi=$ As. Then $\xi \in \operatorname{Ker} A$ (cf. Lemma 3.1(i)) and

$$
2\langle(\operatorname{Re} A) S, S\rangle=\langle A S, S\rangle+\langle S, A S\rangle=\langle\mathrm{T} \xi, S\rangle+\langle S, T \xi\rangle=0
$$

whence $S \in \operatorname{Ker} A$ and therefore $\varepsilon=0$, thus proving (i). Next, suppose $\theta \in \operatorname{Ker} A$ and $\langle\mathrm{T} \theta, \kappa\rangle=0$ for all $\kappa \in \operatorname{Ker} A$. Then $\mathrm{T} \theta \in\left[\operatorname{Ker} \mathrm{A}^{*}\right]^{\perp}=\operatorname{Ran} A$, thus implying $\theta$ $\in K_{0}$. Conversely, if $\theta \in K_{0}$ and $A \lambda=T \theta$, then, for all $\kappa \in \operatorname{Ker} A,\langle T \theta, \kappa\rangle=$ $\langle A \lambda, K\rangle=\left\langle\lambda, A^{*} \kappa\right\rangle=0$, which proves (ii). Part (iii) follows by a simple counting argument. Given maximal positive definite and negative definite subspaces $\mathcal{N}_{ \pm}$of Ker A, we have $\mathcal{N}_{+} \oplus \mathcal{N}_{-} \oplus \mathfrak{K}_{0}=\operatorname{Ker} A$, while $\mathcal{N}_{ \pm} \oplus \mathcal{K}_{0}$ are positive and negative subspaces of $Z_{0}$ whose dimensions add up to the dimension of $Z_{0}$. Since $Z_{0}$ is nondegenerate, we obtain (iii). 0

For later use we define $m_{+}$and $m$.. as the dimensions of a maximal positive definite and a maximal negative definite subspace of $\operatorname{Ker} A$, respectively, and $m_{0}$ as the dimension of the neutral part of Ker A. All three numbers are independent of the particular choice of subspace.

Now recall that $Z_{0} \subseteq D(T)$. Define $\hat{Z}_{0}=T\left[Z_{0}\right], Z_{1}=\left[\hat{Z}_{0}\right]^{\perp}$ and $\hat{Z}_{1}=\left[Z_{0}\right]^{\perp}$. Then Lemma 3.2(i) implies that $Z_{0} \cap Z_{1}=\{0\}$ and $\left.\hat{Z}_{0} \cap \hat{Z}_{1}=10\right\}$. A simple dimension counting argument coupled with some manipulation of orthogonal complements yields $Z_{0} \oplus Z_{1}=\hat{Z}_{0} \oplus \hat{Z}_{1}=H$. Further, if $\varepsilon \in Z_{1}$, then, for all $\eta \in$ 
$Z_{0},\langle A \xi, \eta\rangle=\left\langle\xi, A^{*} \eta\right\rangle=0$ (because $A^{*} \eta \in T\left[Z_{0}\right]$ ), so that $A \xi \in \hat{Z}_{1}$. Thus choosing $\beta$ to be an invertible positive operator on $Z_{0}$ and defining $A_{\beta}$ as the linear operator on $H$ which coincides with $T \beta^{-1}$ on $Z_{0}$ and with $A$ on $Z_{1}$, we may describe $A_{\beta}$ by the following diagram:

$$
\begin{array}{rlll}
Z_{0} \oplus Z_{1} & =H \\
T \beta^{-1} \mid & & A \mid & A_{\beta} \mid \\
\hat{Z}_{0} \oplus & \hat{Z}_{1} & =H
\end{array}
$$

where both decompositions are $\langle\mathrm{T}, \cdot\rangle$-orthogonal. Inner product arithmetic now gives

$$
\left\langle\left(\operatorname{Re} A_{\beta}\right) \xi, \xi>=\left\langle T \beta^{-1} \xi_{0}, \xi_{0}\right\rangle+\left\langle(\operatorname{ReA}) \xi_{1}, \xi_{1}\right\rangle\right.
$$

which implies that $\operatorname{Re} A_{B} \geq \delta 1$ for some $\delta>0$. Here $\xi=\xi_{0}+\xi_{1}$ is the decomposition of $\xi$ according to $Z_{0} \oplus Z_{1}=H$. Then $A_{\beta}$ satisfies condition (1.6), as a result of the additional assumption $Z_{0} \subseteq \mathrm{D}\left(\mid \mathrm{T} l^{\beta}\right)$ for some $\beta>2$.

THEOREM 3.3. Under the general hypotheses of this section, there is at least one solution of Eqs. (1.1)-(1.3) whenever $\varphi_{+} \in Q_{+}[D(T)]$.

Proof: Let us replace Eqs. (1.1)-(1.3) by the boundary value problem

$$
\begin{aligned}
& \left(\mathrm{T} \psi_{\beta}\right)^{\prime}(\mathrm{x})=-\mathrm{A}_{\beta} \psi_{\beta}(\mathrm{x}), 0<\mathrm{x}<\infty, \\
& \mathrm{Q}_{+} \psi_{\beta}(0)=\varphi_{+}, \\
& \left\|\psi_{\beta}(\mathrm{x})\right\|_{\mathrm{H}}=\mathrm{O}(1)(\mathrm{x} \rightarrow \infty),
\end{aligned}
$$

where the maximal $\beta$-invariant subspace $\mathcal{H}$ of $Z_{0}$ such that $\sigma\left(\left.\beta\right|_{\mathcal{M}}\right) \subseteq \mathbf{R}_{+}$is contained in Ker A. According to Lemma 3.2(iii) such a choice of $\beta$ is possible. If $\Pi_{0}$ and $I_{1}$ denote the complementary projections of $H$ onto $Z_{0}$ and $Z_{1}$, respectively, then 


$$
\psi(\mathrm{x})=\Pi_{0} \psi_{\beta}(0)+\Pi_{1} \psi_{\beta}(\mathrm{x})
$$

is a solution of Eqs. (1.1)-(1.3), because $\Pi_{0} \psi_{\beta}(0) \in \operatorname{Ker} A$.

THEOREM 3.4. Under the general hypotheses of this section, the number of linearIy independent solutions of the boundary value problem

$$
\begin{aligned}
& (T \psi)^{\prime}(x)=-A \psi(x), 0<x<\infty, \\
& Q_{+} \phi(0)=0, \\
& \|\psi(x)\|_{H}=o(1) \text {, or } O(1) \text {, or } O(x)(x \rightarrow \infty),
\end{aligned}
$$

is zero in the o(1)-case, $\mathrm{m}_{-}$. In the $O(1)$-case and $\mathrm{m}_{-}+\mathrm{m}_{0}$ in the $\mathrm{O}(\mathrm{x})$-case.

Proof: Following the decomposition procedure given in the proof of Theorem 3.3, we may decompose Eq. (3.4) in an equation on $Z_{1}$ and an equation on $Z_{0}$. The latter has a trivial general solution, which is of the form [cf. Lemma 3.1(ii)]

$$
\psi_{0}(x)=\left[I I-x T^{-1} A\right] \psi_{0}(0)
$$

Thus in the $o(1)$-case we miust have $\psi_{0}(0)=0$, in the $O(1)$-case $\psi_{0}(0) \in \operatorname{Ker} A$ and in the $O(x)$-case $\psi_{0}(0) \in Z_{0}$. In the o(1)-case any solution of Eqs. (3.4)-(3.6) will be a solution of Eqs. (3.1)-(3.3) with $\varphi_{+}=0$. Thus in the o(1)-case Eqs. (3.4)-(3.6) only have the trivial solution [cf. Proposition 2.2 in combination with $\operatorname{Re} A_{F} \geq 0$ ].

Next, consider a solution of Eqs. (3.4)-(3.6) in the $O(x$ )-case (resp. $O(1)$-case). Define

$$
H_{1, p}=H_{p} \cap Z_{1}, \quad H_{1, m}=H_{m} \cap Z_{1}
$$

where $H_{p}$ and $H_{m}$ correspond to Eqs. (3.1)-(3.3). Then $H_{1, p}$ and $H_{1, m}$ are independent of the choice of $\beta$, are positive definite and negative definite, respectively, orthogonal with respect to the $\langle\mathrm{T}, \cdot\rangle$-inner product and add up to all of $Z_{1}$. Exploiting various orthogonality and positivity properties we find a one-to-one correspondence between the solutions of Eqs. (3.4)-(3.6) in the $O(x)$-case (resp. $O(1)$-case) and their $I_{0}$-projections, which must belong to the negative definite subspace $\mathcal{M}_{-}=$ 
$\left[H_{1, p} \oplus H_{\ldots}\right] \cap Z_{0}$ of $Z_{0}$ (resp. $\mathcal{N}_{--}=\left[H_{1, p} \oplus H_{-}\right] \cap \operatorname{Ker} A$ of Ker A). Here $H_{y:}=$ $Q_{ \pm}[H]$. Since obviously the orthogonal complements of $H_{+}, H_{-}, Z_{0}, \operatorname{Ker} A, H_{1, p}$ and $H_{1, m}$ under the $\left\langle T_{r} \cdot\right\rangle-$ inner product are given by the respective subspaces $H_{n}, H_{+}$, $Z_{1}, Z_{1} \oplus \mathcal{K}_{0}, H_{1, m} \oplus Z_{0}$ and $H_{1, p} \oplus Z_{0}$, we find that the $\langle\Gamma, \cdot\rangle$-orthogonal complement of $\mathcal{M}_{-}$in $Z_{0}$ (resp. $\mathcal{N}_{-}$in $\operatorname{Ker} A$ ) is given by $\mathcal{N}_{+}$(resp. $\mathcal{N}_{+} \oplus X_{0}$ ) where $\mathcal{M}_{+}=\left[\mathrm{H}_{1, m} \oplus \mathrm{H}_{+}\right] \cap \mathrm{Z}_{0}$ and $\mathcal{N}_{+}=\left[H_{1, m} \oplus \mathrm{H}_{+}\right] \cap \mathrm{Ker} A$. Because the latter space is positive definite (resp. positive) with respect to the $\langle\Gamma$, $\rangle$-inner product, the original subspace $\mathcal{H}_{\text {.. }}$ (resp. $\mathcal{N}_{-}$) must have been maximal negative definite in $Z_{0}$ (resp. maximal negative definite in Ker $A$ ). Thus $\operatorname{dim} \boldsymbol{A}_{-}=m_{\ldots}+\mathrm{m}_{\mathrm{B}}$ and $\operatorname{dim} \mathcal{N}_{-}=\mathrm{m}_{-}$, which completes the proof of the theorem.

THEOREM 3.5. Under the general hypotheses of this section, the vectors $\varphi_{+} \epsilon$ $Q_{+}[D(T)]$ for which the boundary value problem

$$
\begin{aligned}
& (\mathrm{T} \psi)^{\prime}(\mathrm{x})=-\mathrm{A} \psi(\mathrm{x}), 0<\mathrm{x}<\infty, \\
& \mathrm{Q}_{+} \psi(0)=\varphi_{+}, \\
& \|\psi(\mathrm{x})\|_{\mathrm{H}}=o(1)(\mathrm{x} \rightarrow \infty),
\end{aligned}
$$

has a solution, make up a linear subspace of $\mathrm{Q}_{+}[\mathrm{D}(\mathrm{T})]$ of co-dimension $\mathrm{m}_{+}+\mathrm{m}_{0^{-}}$If a solution exists, it is unlque.

Proof: Given $\varphi_{+} \in Q_{+}[D(T)]$, there is solution of Eqs. (3.8)-(3.10) if and only if $\varphi_{+} \in\left[\mathrm{H}_{1, p}+\mathrm{H}_{-}\right] \cap \mathrm{Q}_{+}[\mathrm{D}(\mathrm{T})]$. Since $\psi(0) \in \mathrm{D}(\mathrm{T})$ also, there is a solution of Eqs. (3.8)-(3.10) if and only if

$$
\varphi_{+} \in\left[H_{1, p}+H_{-} I \cap Q_{+}[D(T)]=\left(H_{1, p} \cap Q_{+}[D(T)]\right)+\left(H_{-} \cap Q_{+}[D(T)]\right)\right.
$$

We now make two observations: (i) $Q_{+}[D(T)]+Q_{-}[D(T)]=D(T)$, and (ii) $\left[H_{1, p} \cap\right.$ $D(T)]+\left[H_{-} \cap D(T)\right]+Z_{0}=D(T)$, the latter following from the existence of a solution of Eqs. (1.1)-(1.3) with (1.3) replaced with $\|\psi(x)\|_{H}=O(x)(x \rightarrow \infty)$ for any $\varphi_{+}$ $\in Q_{+}[D(T)]$. We now apply the linear algebra statement $\mathcal{A}(\mathcal{A} \cap B) \simeq\left(\mathcal{A}+\mathscr{F}^{\mathrm{B}}\right) / \mathrm{B}$ twice, first for $\mathcal{A}=Q_{+}[D(T)]$ and $B=\left[H_{1, p}+H_{\sim}\right] \cap D(T)$ and next for $\mathcal{A}=Z_{0}$ and the same $B$, and conclude that the vectors $\varphi_{+} \in Q_{+}[D(T)]$ for which Eqs. (3.7)(3.10) have a solution, make up a subspace of $Q_{+}[D(T)]$ whose co-dimension coincides 
with the co-dimension of $\mathfrak{H}_{-}=\left[\mathrm{H}_{1, p}+H_{-}\right] \cap Z_{0}$ in $Z_{0}$. Since $\mathbb{M}_{-}$is a maximal negative definite subspace of $Z_{0}$ and $Z_{0}$ is non-degenerate, its co-dimension in $Z_{0}$ must be the same as the dimension of a maximal positive subspace in $Z_{0}$, which equals $m_{+}+m_{0}$. The uniqueness part of this theorem is clear from Theorem 3.4.

The homogeneous boundary value problem (3.4)-(3.6) in the $O(x)$-case, when applied to radiation processes in a stellar atmosphere, is known as the Milne problem (cf. $[\mathrm{Ch}$,Sob]). In fact, in this case we easily obtain that for every e $\in$ Ker $A$

$$
\frac{d}{d x}<T \Downarrow(x), e>=-<A \psi(x), e>=-<\downarrow(x), A^{*} e>=0
$$

which corresponds to the radiative flux being independent of optical depth. Thus, for every e $\in \operatorname{Ker} A, F_{e}(\psi) \stackrel{\text { def. }}{=}\langle T \psi(x), e\rangle$ is a linear functional on the set of Milne solutions. When $e$ is ranging over a basis $\left\{e_{1}, \cdots, e_{m_{-}}\right\}$of some maximal negative definite subspace of $\operatorname{Ker} A$, then for every $\varphi_{+} \in Q_{+}[D(T)]$ there is a unique solution of Eq. (1.1) with boundary conditions (1.2), (1.3) and

$$
F_{e_{j}}(b)=f_{j}=\text { given, }_{j}=1, \cdots, m_{-}
$$

Indeed, if one were to consider the solution of this problem for $\varphi_{+}=0$ and $f_{1}=$ $\cdots=\mathrm{f}_{\mathrm{m}_{-}}=0$, let $\gamma$ be a $<\mathrm{T}$, $>>$-positive invertible operator on $Z_{0}$ mapping the maximal negative subspace $\left[\mathrm{H}_{1, p}+\mathrm{H}_{-}\right] \cap \mathrm{Z}_{0}$ of $\mathrm{Z}_{0}$ onto span $\left\{\mathbf{e}_{1}, \cdots, \mathrm{e}_{\mathrm{m}_{-}}\right\}$. Then $\gamma \psi(\infty)$ can be written as a linear combination of $e_{1}, \cdots, e_{m}$. Since the matrix $\left\{\left\langle T \gamma^{-1} e_{i}, e_{j}\right\rangle\right\}_{i, j=1}^{m_{-}}$is non-singular, we must have $\psi(\infty)=0$ and therefore $\psi(x) \equiv 0$.

\section{APPLICATIONS}

In this section we will apply the theory of Sections 2 and 3 to polarized light transfer and multigroup neutron transport.

\subsection{Polarized light transfer}

The equation of transfer of polarized light in a homogeneous half-space medium has the form (cf. [HM]) 


$$
u \frac{\partial I}{\partial \tau}(u, \tau, \varphi)+I(u, \tau, \varphi)=\frac{a}{4 \pi} \int_{-1}^{1} \int_{0}^{2 \pi} Z\left(u, u^{\prime}, \varphi-\varphi^{\prime}\right) I\left(\tau, u^{\prime}, \varphi^{\prime}\right) d \varphi^{\prime} d u^{\prime}
$$

where $\tau \in(0, \infty)$ is the optical depth (measured from the surface), $(u, \varphi) \in[-1,1] \times$ $[0,2 \pi]$ specifies the direction of propagation of the light, a $\in(0,1]$ is the albedo of single scattering and $I=(I, Q, U, V)$ is the Stokes vector whose first component is the specific intensity and where $Q / I, U / I$ and $V / I$ specify the state of polarization of the light. We impose the boundary conditions

$$
\begin{aligned}
& I(0, u, \varphi)=\mathbb{I}_{+}(\mathrm{u}, \varphi), \quad(\mathrm{u}, \varphi) \in[0,1] \times[0,2 \pi], \\
& \int_{-1}^{1} \int_{0}^{2 \pi}\left\|\mathrm{I}\left(\tau, \mathrm{u}^{\prime}, \varphi^{\prime}\right)\right\|_{2}{ }^{2} \mathrm{~d} \varphi^{\prime} \mathrm{d} \mathrm{u}^{\prime}=\mathrm{O}(1) \quad(\tau \rightarrow \infty),
\end{aligned}
$$

where $\|\mathrm{I}\|_{2}^{2}=\left.\left|I^{2}+\right| \mathrm{Q}\right|^{2}+|U|^{2}+|V|^{2}$. The so-called phase matrix is given by

$$
\mathbf{Z}\left(\mathbf{u}, \mathbf{u}^{\prime}, \varphi-\varphi^{\prime}\right)=\mathbf{L}\left(\pi-\sigma_{2}\right) \mathbf{F}(\theta) \mathbf{L}\left(-\sigma_{1}\right)
$$

with the scattering matrix $F(\theta)$ and the rotation matrix $L(\alpha)$ given by

$$
\mathbf{F}(\theta)=\left[\begin{array}{cccc}
a_{1}(\theta) & b_{1}(\theta) & 0 & 0 \\
b_{1}(\theta) & a_{2}(\theta) & 0 & 0 \\
0 & 0 & a_{3}(\theta) & b_{2}(\theta) \\
0 & 0 & -b_{2}(\theta) & a_{4}(\theta)
\end{array}\right]
$$

and

$$
\mathbf{L}(\alpha)=\left[\begin{array}{cccc}
1 & 0 & 0 & 0 \\
0 & \cos 2 \alpha & \sin 2 \alpha & 0 \\
0 & -\sin 2 \alpha & \cos 2 \alpha & 0 \\
0 & 0 & 0 & 1
\end{array}\right] \text {, }
$$

respectively, and $\varphi^{\prime}-\varphi, \sigma_{1}$ and $\sigma_{2}$ forming the angles and $\theta, \vartheta$ and $\vartheta^{\prime}$ the opposite sides of a spherical triangle, where $u=-\cos \vartheta$ and $u^{\prime}=-\cos \vartheta^{\prime}$. The scattering matrix consists of Borel functions of $\theta$ with $a_{1}(\theta)$ nonnegative with $\int_{-1}^{1} a_{1}(\theta) d(\cos \theta)$ $=2$ and leaves invariant the cone of real vectors 1 satisfying $I \geq\left(Q^{2}+U^{2}+V^{2}\right)^{1 / 2}$ for almost every $\theta \in[0, \pi]$. As a result, on choosing $H$ to be the (complex) Hilbert space of vector functions $\mathbb{I}:[-1,1] \times[0,2 \pi] \rightarrow \mathbb{C}^{4}$ with the usual $L_{2}$-norm, we find $(T I)(u, \varphi)=u I(u, \varphi)$ to be bounded, injective and self-adjoint on $H$ and $(A I)(u, \varphi)=$ 
$\mathbb{I}(u, \varphi)-(\mathrm{a} / 4 \pi) \int_{-1}^{1} \int_{0}^{2 \pi} Z\left(u, u^{\prime}, \varphi-\varphi^{\prime}\right) \amalg\left(u^{\prime}, \varphi^{\prime}\right) \mathrm{d} \varphi^{\prime} d u^{\prime}$ to be a compact perturbation of the identity satisfying $\operatorname{Re} A \geq 0$ and $\operatorname{Ker} A=\operatorname{Ker}(\operatorname{Re} A)$. If $a<1$, we have $\operatorname{Re} A$ $z \delta 1$ for $\delta=1-a>0$, while for $a=1$ we have $\operatorname{dim} \operatorname{Ker} A=1$ and $\operatorname{dim} Z_{0}=2$, except for the exceptional case $a_{1} \equiv a_{4}$ where these dimensions are 2 and 4 . For the statement on the nonnegativity of Re A we refer to Section IX.2 of [GMP]; for the statements on the structure of $Z_{0}$ we refer to [M4]. Moreover, condition (1.6) is satisfied if $a_{1} \in L_{r}[-1,1]$ for some $r>1$ (see [GMP], Sec. IX.2). From Theorem 3.4 it is then clear that Eqs. (4.1)-(4.3) are uniquely solvable when stated on the Hilbert space $H$, provided $a_{1} \in L_{r}[-1,1]$ for some $r>1$. In this way we recover the result of $[\mathrm{M} 4]$ for the $L_{2}$-setting, but without applying cone preservation arguments as in [M4].

\subsection{Multigroup neutron transport}

For isotropic scattering and a homogeneous half-space medium the N-group neutron transport equation has the form (cf. [DuM], [CaZ])

$$
\mu \frac{\partial \psi_{i}}{\partial \mathrm{x}}(\mathrm{x}, \mu)+\sigma_{i} \xi_{i}(\mathrm{x}, \mu)=\frac{1}{2} \sum_{j=1}^{\mathrm{N}} c_{i j} \int_{-1}^{1} \psi_{j}\left(\mathrm{x}, \mu^{\prime}\right) \mathrm{d} \mu^{\prime},
$$

where $x \in(0, \infty)$ is the distance from the surface (in units of the largest mean free path among the $\mathrm{N}$ energy groups) and $\mu$ is the direction cosine of propagation. The matrix $\mathcal{C}=\left\{c_{t j}\right\}{ }_{i, j=1}^{N}$ has nonnegative elements only and $\sigma_{1}>\cdots>\sigma_{N}>0$. We use the short-hand notation $\Sigma=\left\{\sigma_{i} \delta_{i j}\right\}_{i, j=1}^{N}$ and impose the boundary conditions

$$
\begin{aligned}
& \psi_{i}(0, \mu)=\varphi_{i}(\mu), \quad \mu \in[0,1] \\
& {\left[\int_{-1}^{1}\left|\psi_{i}\left(x, \mu^{\prime}\right)\right|^{2} \mathrm{~d} \mu^{\prime}\right]^{1 / 2}=\mathrm{O}(1) \quad(x \rightarrow \infty),}
\end{aligned}
$$

where $i=1, \cdots, N$. We write $\psi=\operatorname{col}\left\{\psi_{i}\right\}_{i=1}^{N}$ and $\Phi=\operatorname{col}\left\{\varphi_{i}\right\}_{i=1}^{N}$. One gets an application of Sections 2 and 3 by defining the operators $(\mathrm{T} \Psi)_{i}(\mu)=\sigma_{i}^{-1} \mu \psi_{i}(\mu)$ and $(A \psi)_{1}(\mu)=\psi_{i}(\mu)-\frac{1}{2}\left[\Sigma^{-1} \mathrm{C} \int_{-1}^{1} \psi\left(\mu^{\prime}\right) \mathrm{d} \mu^{\prime}\right]_{i}$. Then $\mathrm{T}$ is bounded, injective and selfadjoint and $A$ is a compact perturbation of the identity satisfying (1.6), both defined on the Hilbert space $H$ given as the weighted $L_{2}$-direct sum of $N$ copies of $L_{2}[-1,1]$ with weights $\sigma_{1}, \cdots, \sigma_{N^{*}}$

Define $\mathscr{B}$ on $\mathbb{C}^{\mathrm{N}}$ as $\Sigma^{-1} \mathrm{C}, A: \mathrm{H} \rightarrow \mathbb{C}^{\mathrm{N}}$ by $(A \Psi)_{i}=\frac{1}{2} \int_{-1}^{1} p\left(\mu^{\prime}\right) \mathrm{d} \mu^{\prime}$ and $\mathrm{K}_{0} \mathbb{C}^{\mathrm{N}} \rightarrow$

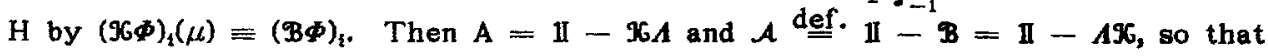


$A$ and $\mathcal{A}$ have the same nonzero eigenvalues. Moreover, $\operatorname{Re} \mathrm{A} \geq 0$ if and only if $\operatorname{Re}(\Sigma-C) \geq 0$, while $\operatorname{Ker} A=\operatorname{Ker}(\operatorname{Re} A)$ holds true if and only if $\operatorname{Ker} \Sigma-C=$ $\operatorname{Ker}(\operatorname{Re}(\Sigma-C))$. We may then apply the theory of Sections 2 and 3 and derive some existence and uniqueness results. In particular, if $\left\|\Sigma^{-1} \mathrm{C}\right\|<1$ in the Euclidean norm of $\mathbb{C}^{N}$, then the boundary value problem (4.4)-(4.6) is uniquely solvable. The last result was found before by Bowden et al. [BSZ] using "Caseology".

\section{DISCUSSION}

A number of topics has not been treated in Sections 2 and 3, for the simple reason that we did not need them to prove the (non-) unique solvability of the main boundary value problem. In order to fit in our work with some of the previous research, we will briefly outline these topics.

First of all, under the general assumptions of Section 3 , the subspaces $H_{1, p}$ $H_{1, \pi}$ and $Z_{0}$ are invariant under $T^{-1} A$ and the restrictions of $T^{-1} A$ to these subspaces have their spectrum in $\{\operatorname{Re} \lambda>0\} \cup\{0\},\{\operatorname{Re} \lambda<0\} \cup\{0\}$ and $\{0\}$, respectively; only the restriction to $Z_{0}$ has eigenvalue spectrum at zero. Moreover, the \pm restrictions of $T^{-1} A$ to $H_{1, p}$ and $H_{1, m}$ generate bounded analytic semigroups which are strongly vanishing as $x \rightarrow \infty$. One way to prove it proceeds by estimating the resolvent of $T^{-1} A$ restricted to these subspaces and applying the appropriate result from semigroup theory.

Secondly, it is possible to drop the regularity assumption (1.6) from the theory of this paper. Instead we have to assume (i) Ran $B \subseteq D(T)$ and (ii) $Z_{0} \subseteq D\left(T^{2}\right.$ ). Basically, all we need condition (1.6) for is for the operators $\mathcal{L}_{\mathrm{B}}$ and $\mathcal{L}_{\mathrm{TB}}$ to be bounded on $\mathrm{C}(\mathrm{H})_{0}^{\infty}$. This will be shown in a future publication (cf. [GM]).

\section{REFERENCES}

B1. R. Beals, "On an abstract treatment of some forward-backward problems of transport and scattering," J. Funct. Anal. 34, 1-20 (1979).

B2. R. Beals, "Indefinite Sturm-Liouville problems and half-range completeness," J. Diff. Eqs. 56, 391-407 (1985).

Bo. J. Bognár, "Indefinite inner product spaces," Berlin, Springer, 1974.

BSZ. R.L. Bowden, S. Sancaktar and P.F. Zweifel, "Multigroup neutron transport. II. Half-range," J. Math. Phys. 17, 82-86 (1976). 
CaZ. K.M. Case and P.F. Zweifel, "Linear transport theory," Reading, Mass., Addison-Wesley, 1967.

Ch. S. Chandrasekhar, "Radiative transfer," London, Oxford Univ. Press, $1950=$ New York, Dover Publ., 1960.

DuM. J.J. Duderstadt and W.R. Martin, "Transport theory," New York, Wiley-Interscience, 1979.

Fe3. I.A. Feldman, "On some projection methods for the solution of the equation of radiative energy transfer," Matem. Issled. 7 (4), 228-236 (1972) [Russian].

Fe. I.A. Feldman, "On Wiener-Hopf equations with weakly integrable operator kernels," Matem. Issled. 8 (4), 101-110 (1973) [Russian].

Ga. A.H. Ganchev, Ph.D. Thesis, Dept. of Mathematics, Virginia Tech, 1986.

GaG. A.H. Ganchev and W. Greenberg, "Linear half-space transport equations with accretive, invertible collision operators," Transp. Theory Stat. Phys., to appear.

GaGM. A.H. Ganchev, W. Greenberg and C.V.M. van der Mee, "A class of linear kinetic equations in a Krein space setting," Int. Eqs. Oper. Theory, submitted.

GK. I.C. Gohberg and M.G. Krein, "Systems of integral equations on a half-line with kernels depending on the difference of arguments," Amer. Math. Soc. Transl., Series 2, 14, 217-287 (1960) = Uspehi Matem. Nauk 13 (2), 3-72 (1959) [Russian].

GLR. I. Gohberg, P. Lancaster and L. Rodman, "Matrix functions and indefinite scalar products," Basel and Boston, Birkhäuser OT 8, 1983.

GL2. I.C. Gohberg and J. Leiterer, "Factorization of operator functions with respect to a contour. II. Canonical factorization of operator functions close to the identity," Math. Nachrichten 54, 41-74 (1972) [Russian].

GM. W. Greenberg and C.V.M. van der Mee, "Generalized kinetic equations with nonregular collision operators," In preparation.

GMP. W. Greenberg, C.V.M. van der Mee and V. Protopopescu, "Boundary value problems in abstract kinetic theory," Basel and Boston, Birkhäuser OT 23, 1987.

GMW. W. Greenberg, C.V.M. van der Mee and W. Walus, "Strong solutions for abstract kinetic equations with compact collision operators," Int. Eqs. Oper. Theory, to appear.

GMZ. W. Greenberg, C.V.M. van der Mee and P.F. Zweifel, "Generalized kinetic equations," Int. Eqs. Oper. Theory 7, 60-95 (1984).

H. R.J. Hangelbroek, "Linear analysis and solution of neutron transport problems," Transp. Theory Stat. Phys. 5, 1-85 (1975).

HL. R.J. Hangelbroek and C.G. L.ekkerkerker, "Decompositions of a Hilbert space and factorization of a W-A determinant," SIAM J. Math. Anal. 8, 458-472 (1977).

HM. J.W. Hovenier and C.V.M. van der Mee, "Fundamental relationships relevant to the transfer of polarized light in a scattering atmosphere," Astron. Astrophys. $128,1-16$ (1983).

KLH. H.G. Kaper, C.G. Lekkerkerker and J. Hejtmanek, "Spectral methods in linear transport theory," Basel and Boston, Birkhäuser OT 5, 1982.

Le. C.G. Lekkerkerker, "The linear transport equation. The degenerate case $\mathrm{c}=1$. I. Full-range theory; II. Half-range theory," Proc. Roy. Soc. Edinburgh 75A, 259-282 and 283-295 (1975/76).

M1. C.V.M. van der Mee, "Semigroup and factorization methods in transport theory," Ph.D. Thesis, Free University, Amsterdam, 1981.

M3. C.V.M. van der Mee, "Albedo operators and H-equations for generalized kinetic models," Transp. Theory Stat. Phys. 13, 341-376 (1984).

M4. C.V.M. van der Mee, "Half- and finite-range completeness for the equation of 
transfer of polarized light," Math. Meth. Appl. Sci. 6, 393-416 (1984).

Sob. V.V. Sobolev, "Light scattering in planetary atmospheres," Oxford, Pergamon Press, 1975 = Moscow, Nauka, 1972 [Russian].

WZM. B.L. Willis, P.F. Zweifel and C.V.M. van der Mee, "Whither existence theory?," Transp. Theory Stat. Phys. 14, 669-678 (1985).

\author{
William Greenberg \\ Dept. of Mathematics, and \\ Center for Transport Theory and Mathematical Physics \\ Virginia Polytechnic Institute and State University \\ Blacksburg, Virginia 24061
}

\author{
Alexander H. Ganchev \\ C.V.M. van der Mee \\ Bulgarian Academy of Sciences / INRNE \\ Dept. of Mathematics and Statistics \\ Blvd. Lenin 72 \\ University of Pittsburgh \\ Sofia 1784, Bulgaria \\ Pittsburgh, Pennsylvania 15260
}

Submitted: January 20, 1986

Revised: December 10, 1987 